\title{
ESTUDO PALEOAMBIENTAL PRELIMINAR DO CRETÁCEO DA BACIA POTIGUAR
}

\author{
MARTA CLẢUDIA VIVIERS* e MARÍLIA DA SILVA PARES REGALI**
}

\begin{abstract}
ABETRACT Integration of micropaleontological information, based mainly on the study of foraminifers and palynomorphs from seven wells, led to the recognition of the paleoenvironmental development of the Potiguar Basin from the Alagoas to the Maastrichtian. Seventeen bioestratigraphical zones and one subzone have been recognized. Seven episodes - four transgressive and three regressive - could be detected between the Early-Middle Albian to the Maastrichtian, and have been regarded as result from relative global fluctuations of the Cretaceous sea level. A distinctive hiatus of Late Albian age may be inferred from the absence of diagnostic index fossils. Fine grained clastic sediments deposited during the Late Cenomanian and the Early Turonian show evidence of anoxia. This is assumed to be synchronous with similar events detected elsewhere in many Cretaceous basins (including some in Brazil). Carbonatic cycles have been found to occur in the Early-Middle Albian, Early-Middle Cenomanian, Turonian and Coniacian/Santonian, each of them being represented by shallow neritic paleoecological units with distinctive microfaunal assemblages: Early-Middle Albian - Favusella spp., calcispherulids association; Early-Middle Cenomanian - Trocholina spp. association; Turonian - marine ostracods-gastropods-worm tubes association; Santonian - Buliminidae-Siphogenerinoides spp.-Dinogymnium spp.-Deflandrea spp. association.
\end{abstract}

INTRODUÇÃo Desenvolve-se atualmente, no Centro de Pesquisas da Petrobrás, um projeto que investiga a evolução da Bacia Potiguar, durante o Meso-Neocretáceo (Alagoas-Eomaastrichtiano). Este trabalho apresenta, preliminarmente, informações bioestratigráficas e paleoecológicas colhidas ao longo de una seção aproximadamente perpendicular à linha de costa, composta pelos peços 1-PT-1, 1-RNS-36, 1-RNS-50， 3-RNS-38， 4-RNS-35, 1-RNS-25 e 1-RNS-3 (Fig. 1).

A integração de dados micropaleontológicos foi feita com o objetivo de se obter um conhecimento mais completo da história evolutiva deste sítio sedimentar. Buscou-se refinar o arcabouço bioestratigráfico, reconhecer a sucessão de unidades paleoecológicas e elaborar modelos paleoambientais integrados, a partir de dados de palinomorfos, foraminíferos e outros microfósseis.

MÉTODO DE TRABALHO Amostras de calha e testemunhos foram analisados pelos métodos qualitativo e semiquantitativo. Os foraminíferos bentônicos, dinoflagelados e acritarcas foram utilizados como indicadores paleoambientais enquanto foraminíferos planctônicos e pólens serviram, principalmente, para fins bioestratigráficos. As amostras de calha foram estudadas a intervalos regulares de $30 \mathrm{~m}$. Os testemunhos disponíveis também foram investigados, tomando-se uma amostra a cada metro testemunhado.

Utilizaram-se, como referência, os zoneamentos palinológicos e de foraminíferos planctônicos propostos por Regali et al. (1974, 1985), Regali (1980) e Viviers (1982, 1985), respectivamente. As unidades bioestratigráficas referem-se a zonas de intervalo.

BIOESTRATIGRAFIA A sucessão de biozonas, suas relações e a amplitude das formas-guias usadas para definir as unidades encontram-se na figura 2 . Por meio de foraminí-

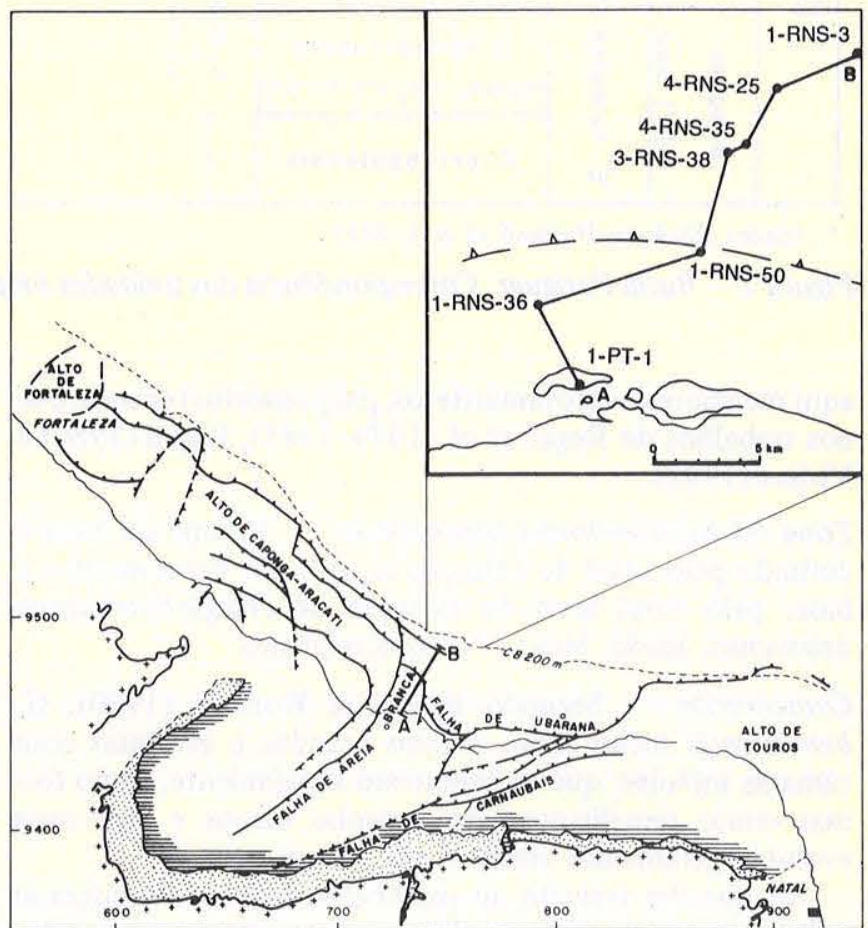

Figura 1 - Bacia Potiguar. Mapa de localização da seção analisada (modificado de Souza 1982)

feros planctônicos e palinomorfos foram reconhecidas quatro superzonas, 17 zonas e uma subzona. Duas zonas e a subzona são novas: as primeiras baseiam-se em foraminíferos planctônicos e a última é caracterizada por um gênero de foraminífero bentônico. Formalizam-se aqui as novas unidades, ao passo que a descrição de todas as outras zonas

* Centro de Pesquisas da Petrobrás (Cenpes), Setor de Bioestratigrafia e Paleoecologia. Cidade Universitária, Quadra 7, Prédio 20, Ilha do Fundão, CEP 21910, Rio de Janeiro, RJ, Brasil

** Centro de Pesquisas da Petrobrás (Cenpes), Divisão de Ensino. Praça Mahatma Gandhi, 14, 140 andar, CEP 20031, Rio de Janeiro, RJ, Brasil 


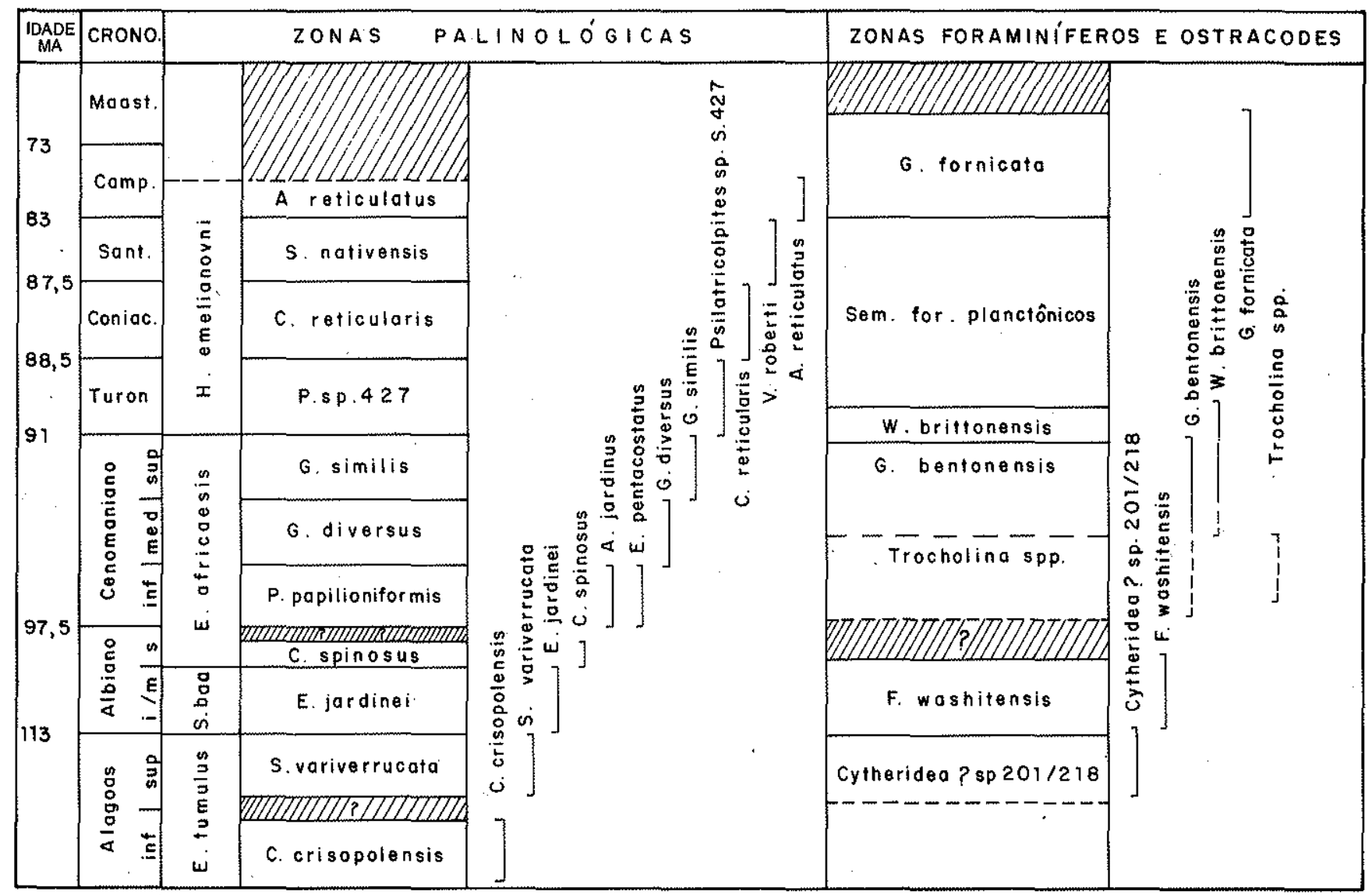

* Idades absolutas Harland et al. (1982)

Figura 2 - Bacia Potiguar. Correspondência das unidades bioestratigráficas

aqui mencionadas (foramin íferos, palinomorfos) encontra-se nos trabalhos de Regali et al. $(1974,1985)$, Regali (1980) e Viviers (1982).

Zona Globigerinelloides bentonensis $O$ topo da zona é definido pelo nível de extinção local de $G$. bentonensis e a base, pelo nível local de extinção de Praeglobotruncana delrioensis. Idade: Meso a Neocenomaniano.

Comentários Segundo Eicher \& Worstell (1970), G. bentonensis inclui tanto formas grandes e involutas com câmaras infladas, que se avolumam rapidamente, como formas muito semelhantes, de tamanho menor e algo mais evolutas, geralmente similares a $G$. caseyi.

No que diz respeito ao material estudado, considera-se difícil separar as duas formas, sendo que, por outro lado, ambas se extinguem no mesmo intervalo estratigráfico. Eicher (1969) assinala que $G$. bentonensis se extingue no topo do Cenomaniano; também Petters (1977) menciona que esta espécie, assim como $G$. caseyi, extingue-se na Zona Rotalipora cushmani (Neocenomaniano). $\mathrm{Na}$ Bacia Potiguar, o nível de desaparecimento de $G$. bentonensis está estratigraficamente bem acima do nível de extinção de $P$. delrioensis e de esporádicas rotaliporas. $\mathrm{Na}$ Bacia do Ceará, também é encontrada a mesma seqüência de eventos bioestratigráficos. Da mesma forma, em ambas as bacias, o topo da Zona $G$, bentonensis coincide aproximadamente com - topo da Zona Gnetaceapollenites similis, que, com a extinção da forma-guia $G$. similis, marca o fim do Cenomaniano nas bacias da margem equatorial brasileira (Regali \& Gonzaga 1985, Regali et al. 1985).
Zona Whiteinella brittonensis 0 topo da zona é definido pelo nível de extinção local de $W$. brittonensis e a base, pelo nível local de extinção de $G$. bentonensis. Idade: Neocenomaniano-Eoturoniano.

Comentários Esta é uma das zonas melhor e mais comumente mencionada em todos os zoneamentos do Cretáceo. Corresponde à Zona "Grandes Globigerines seules" de Lehmann (1963) e também à Zona Whiteinella archaeocretacea de Robaszinski \& Caron (1979) e Wonders (1980).

Este é o intervalo bioestratigráfico bem definido entre o desaparecimento do gênero Rotalipora e o aparecimento de Praeglobotruncana helvetica.

Em quase todos os zoneamentos conhecidos, nesta zona é mencionada uma renovação muito importante na fauna de foraminiferos planctônicos, correspondendo a um intervalo onde predominam as formas hedbergeliformes grandes e pustulosas (Hedbergella e Whiteinella), e aparecem evolutivamente as primeiras formas dicarinadas (Wonders 1980). Segundo este autor, é uma zona que não goza de populari. dade porque a associação de formas planctônicas presentes no intervalo não é considerada diagnóstica.

W. brittonensis, forma-guia da zona, é comumente mencionada como desaparecendo no Santoniano (Hart et al. 1981). Entretanto, nas bacias da costa brasileira (Ceará, Potiguar e Campos), esta espécie não ocorre com espécies planctônicas características do Coniaciano ou Santoniano.

Em Zaire e Cabinda, na costa ocidental africana, Seiglie \& Baker (1982) definem a Zona $W$. brittonensis da mesma 
forma e a posicionam no Meso-Neocenomaniano/Turoniano basal, podendo-se, portanto, estabelecer uma boa correlação entre estas biozonas definidas nas margens opostas do Atlântico Sul.

Subzona Trocholina ssp. Esta unidade corresponde ao intervalo caracterizado pela amplitude local de Trocholina spp. Idade: Eo a Mesocenomaniano (?)

Comentários Esta subzona está amplamente representada na Bacia Potiguar, sendo que o elemento mais carac. terístico do intervalo é o foraminífero bentônico Trocholina spp., comumente relacionado com os depósitos calcários da Formação Ponta do Mel.

A idade Eo-Mesocenomaniano é estabelecida pelas relações estratigráficas com a Zona Globigerinelloides bentonensis e com os niveis de extinção dos palinomorfos $P$ silatricolpites papilioniformis e Gnetaceapollenites diversus, formas-guia das zonas homônimas, que, nas bacias da margem equatorial brasileira, marcam o Eo-Mesocenomaniano (Regali et al. 1985) (Fig. 2).

\section{ZONEAMENTOS E RELACÓES CRONOESTRATIGRÁ-}

FICAS A integração dos dados bioestratigráficos permitiu conhecer a evolução cronoestratigráfica da seção investigada (Fig. 3).

Nas camadas inferiores da área estudada, foram reconhecidas a Zona Clavatipollenites crisopolensis (Alagoas Inferior) e as zonas correlativas Sergipea variverrucata e Cythe. ridea? sp. 201/218 (Alagoas Superior), caracterizadas por palinomorfos e ostracodes.
Ao Albiano, parte inferior (sensu lato) correspondem as zonas Favusella washitensis e Elateropollenites jardinei. O Albiano Superior (parte inferior) contém a Zona Classopollis spinosus, pouco representada na seção analisada. A falta da Zona Ticinella primula e da Zona Elaterosporites protensus evidenciaria a existência de um hiato que abrangeria pelo menos a parte alta do Neoalbiano.

O Eocenomaniano é representado pela Zona Psilatricopites papilioniformis e sua parte média pela Zona Gnetaceapollenites diversus.

A amplitude local de Trocholina spp. é relacionada com ambas as zonas polínicas. No Neocenomaniano o nível de desaparecimento local de Gnetaceapollenites similis coincide aproximadamente com o de Globigerinelloides bentonensis, ambas formas-guia das zonas homônimas.

De um modo geral, na área proximal da seção (poços 1-PT-1, 1-RNS-36 e 1-RNS-50), as zonas de palinomorfos do Cenomaniano ( $P$. papilioniformis, $G$. diversus e $G$. similis) estão relacionadas com foraminíferos bentônicos aglutinantes (Reophax sp., Thomasinella punica e Cribratina sp.). Esta associação de foraminiferos bentônicos é amplamente conhecida para o Cenomaniano do Mar de Tethys Ocidental, costa norte-oriental da América do Sul e costa ocidental africana (Gargouri 1982, Arnaud-Vanneau \& Prestat 1984).

Os sedimentos turonianos são reconhecidos pela Zona Whiteinella brittonensis na parte inferior (limite Cenomaniano/Turoniano) e pela Zona Psilatricolpites sp. S. 427, na parte superior.

O Cretáceo Superior marinho, na área estudada, é de idade Coniaciano a Eomaastrichtiano, caracterizado pelas

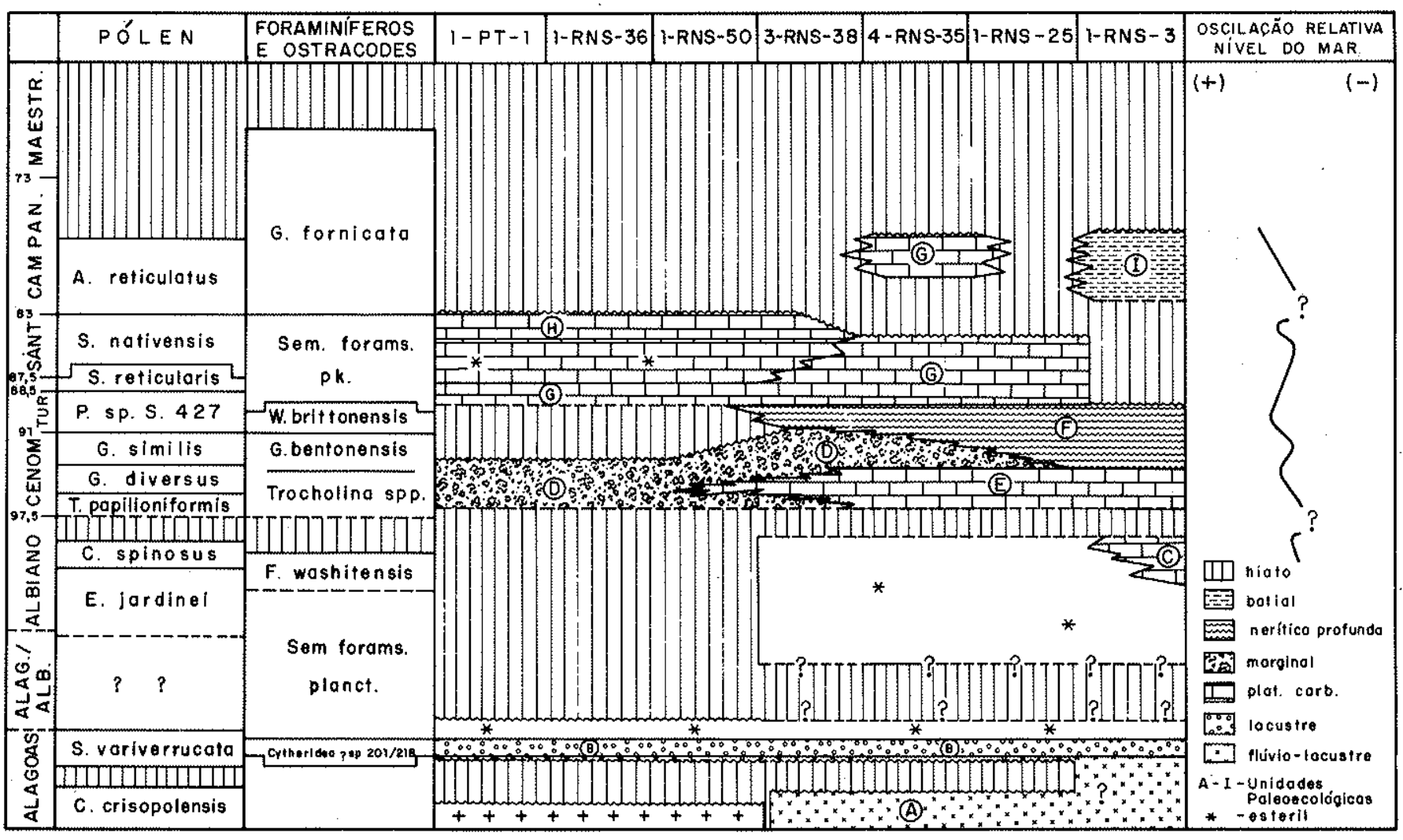

Figura 3 - Evoluçäo paleoecológica das unidades cretáceas na Bacia Potiguar. Area dos poços 1-PT-1, 1-RNS-36, 1-RNS-50, 3-RNS-38, 4-RNS-35, 1-RNS-25 e 1-RNS-3 
Zonas Cupanieidites reticularis/Steevesipollenites nativensis Coniaciano-Santoniano) e Auriculitides reticulatus (Eocampaniano) e Globotruncana fornicata (Campaniano-Eomaastrichtiano). Os níveis de idade campaniana aparecem em forma descontínua na seção estudada (Fig. 3).

FALEOAMBIENTES A seqüência sedimentar, com seus grupos fauniflorísticos distribuídos no tempo e no espaço, foi subdividida em unidades paleoecológicas (UP). A historia evolutiva dessas unidades é apresentada na figura 3.0 quadro 1 contém os conjuntos bióticos que caracterizam cada unidade paleoccológica e as correspondentes unidades litoestratigráficas em que estão representadas.

Os dados estratigráficos foram obtidos da atualização litoestratigráfica da Bacia Potiguar realizada por Souza (1982).

Quadro 1 - Unidades paleoecológicas: c, comum; e, escasso

A. Ambiente: flúvio-lacustre

Conchostráceos

Pólen

Idade: Eo-Alagoas

Formação: Alagamar

B. Ambiente: lacustre

Cytheridea? sp. 201/218(c)

Bisulcocypris sp. (e)

Idade: Neo-Alagoas

Formação: Alagamar

Camadas Ponta do Tubarão

C. Ambiente: nerítico raso (plataforma carbonática incipiente)

Favusella washitensis ( $e$ )

Aracajuia benderi $(e)$

Nodosarídios $(e)$

Textularídios $(e)$

Micromoluscos $(e)$

Phitonella sphaerica $(c)$

Idade: Albiano

Tecas quitinosas de foraminíferos

Dinoflagelados de espinhos curtos

Formação: Ponta do Mel (parte inferior)

D. Ambiente: nerítico raso (marginal-deltaico)

Reophax sp. (c)

Cribatina sp. (e)

Thomasinella punica $(e)$

Brachycythere sapucariensis (c)

Ostracodes (c)

Triorites africaensis

Pemphixipollenites inequiexinius

Odontochitina sp.

Florentinia sp.

Cyclonephelium sp.

Idade: Cenomaniano

Gonyaulax sp.

E. Ambiente: nerítico raso (plataforma carbonática)

Trocholina sp. (c)

Brachycythere sapucariensis $(e)$

Restos de equinóides

Idade: Eo-Mesocenomaniano?

Spiniferites ramosus

Dinoflagelados de espinhos curtos

Formação: Açu

Ambiente: nerítico raso a profundo (marinho aberto)

Whiteinella aprica

Whiteinella baltica

Whiteinella archaeocretacea

Idade: Neocenomaniano

Eoturoniano

Cyclonephelium $\mathrm{sp}$.

Algas verdes e vermelhas

Formação: Ponta do Mel (parte superior)

G. Ambiente: nerítico raso (plataforma carbonática rasa)

Ostracodes marinhos $(c)$

Gastrópodes (c)

Tubos de vermes $(c)$

Espinhas de equinóides $(e)$

Hedbergella delrioensis

Heterohelix spp.

Globigerinelloides bentonensis

Algas verdes

Miliolídeos $(e)$

Brachycythere sapucariensis $(c)$

Idade: Turoniano-Campaniano

Formação: Ubarana

Mb. Quebradas

H. Ambiente: nerítico raso (plataforma carbonática rasa, com influência de água doce)

Buliminella spp. (c)

Afrobolivina afra (c)

Siphogenerinoides clavata $(c)$

Siphogenerinoides bramletti $(c)$

Siphogenerinoides dentata $(c)$

hilum sp. $(e)$

Cyclonephelium

Dino $\mathrm{X}(c)$

Florentinia sp.

Lenticulina sp. $(c)$

Brachycythere sp. $(e)$

Idade: Santoniano

Formação: Jandaíra (parte superior)

I. Ambiente: talude

Bathysiphon sp. (c)

Clavulina sp. (c)

Ammodiscus sp. (c)

Dorothia sp. (c)

Rzehakina sp. $(e)$

Idade: Campaniano-Eomaastrichtiano?

Dinogymnium nelsonense $(c)$

Dinogymnium undulatum $(c)$

Deflandrea sp. (c)

Azolla sp. (c)

Formação: Ubarana 
Alagoas UNIDADE PALEOECOLOGICA A (UPA) A base da seção estudada é composta por sedimentos acumulados em ambiente flúvio-lacustre, conforme sugere a associação de conchostráceos, formas típicas de ambiente continental. Tal deposição ocorreu na parte inferior do Alagoas.

UNIDADE PALEOECOLOGICA B (UPB) - Na parte superior do Alagoas, o sistema evoluiu para um ambiente lacustre relativamente estável do ponto de vista tectônico, com níveis ricos em ostracodes dos gêneros Cytheridea? e Bisulcocypris. A baixa diversidade que apresenta a micro. fauna sugere condições estressantes no meio deposicional.

Albiano UNIDADE PALEOECOLOGICA C (UPC) A instalação marinha, na área estudada, deu-se a partir do Eo-Mesoalbiano, caracterizada por camadas com Favusella washitensis, Aracajuia benderi, escassos restos de gastrópodes, dinoflagelados de espinhos curtos e tecas quitinosas de foraminíferos. Estudos microbiofaciológicos realizados por Dias-Brito (1985) revelam a existência de níveis ricos em calcisphaerulidae, em especial Pithonella sphaerica, além de escassos foraminiferos bentônicos. Esta unidade paleoecológica depositou-se em meio nerítico raso e sob condições adversas para a vida bentônica, o que é evidenciado pela escassez de elementos deste habitat.

Cenomaniano Seguem-se camadas de idade cenomaniana que apresentam uma distribuição de associações microfaunísticas de palinomorfos bem mais rica e complexa que as anteriores. Os sedimentos desta idade foram depositados em um ambiente generalizado de plataforma, com predomínio de sedimentação carbonática durante o Eo-Mesocenomaniano (?) e terrígena durante o Meso-Neocenomaniano.

Em conseqüência, durante o Cenomaniano, podem ser individualizadas várias unidades paleoecológicas que se implantaram como resposta tanto a condiçōes físico-químicas do meio como a variações relativas do nível do mar e a influência da magnitude do aporte sedimentar proveniente do continente. São elas:

UNIDADE PALEOECOLOGICA D (UPD) - Durante todo o Cenomaniano, a área dos poços 1-PT-1 a 1-RNS-38 fez parte de um sistema marginal, compreendido entre a costa e a plataforma carbonática. Os componentes bióticos desta unidade apresentam variações relativas, em estreita vinculação com o volume do aporte continental recebido. Assim, durante o Eocenomaniano, a associação micropaleontológica caracteriza-se por uma fauna pouco desenvolvida de foraminíferos bentônicos aglutinantes (Reophax sp., Thomasinella punica, Cribratina sp.) miliolídeos, restos de gastrópodes, equinóides, ostracodes marinhos, dinoflagelados de espinhos curtos e grande freqüência de Classopollis sp. (pólen de coníferas com grande preferência por proximidade de costa e terrenos salinos - Hughes \& Moody-Stuart 1967, Vakhrameev 1981). A abundância de pólen e a composição da fauna bentônica estariam indicando uma área nerítica bem marginal, com profundidades provavelmente inferiores a $10 \mathrm{~m}$.

Associações de foraminíferos bentônicos dos gêneros acima mencionados tiveram grande expansão em área durante o Cenomaniano, constituindo biocenose típica de ambientes relativamente calmos, nas cercanias de áreas costeiras e na periferia de plataformas carbonáticas (Gargouri 1982).
Já durante o Mesocenomaniano, na mesma área, esta associação fauniflorística apresenta-se qualitativa e quantitativamente mais rica, acrescentando-se a participação dos dinoflagelados Odontochitina sp., Cyclonephelium sp., Gonyaulax sp., Florentinia sp., Spiniferites ramosus e vários dinoflagelados de espinhos curtos. A riqueza deste grupo e a fauna de foraminíferos bentônicos aglutinantes, tão pouco diversificada como no intervalo anterior, embora mais desenvolvida, evidenciam que, durante a metade do Cenomaniano, a influência marinha foi mais acentuada que durante o Eocenomaniano.

No Neocenomaniano, a UPD, restrita aos poços 1-RNS-50 e 3-RNS-38, continua sendo representada pelos foraminiferos aglutinantes, ostracodes etc., além de grande abundância de pólen, principalmente de Classopollis sp., Triorites africaensis, Pemphixipollenites inequiexinius. Novamente, nesses níveis ricos em pólen, os dinoflagelados estão ausentes. A falta desses elementos indicaria alguma modificação na salinidade das águas, causada pela magnitude do aporte continental.

Embora os organismos mais constantes desta unidade paleoecológica sejam os foraminíferos aglutinantes, a razão pólen/dinoflagelado é o indice que melhor mostra as variações ambientais acontecidas na área marginal da plataforma carbonática. Assim, durante o Mesocenomaniano, a presença de Spiniferites ramosus (dinoflagelado com preferência por mar aberto), bem como a diversificação dos dinoflagelados, indicaria que as condições marinhas não foram muito restritas.

Já os níveis do Eo-Neocenomaniano, com grande freqüência de pólen e sem dinoflagelados, poderiam indicar forte influência de ambiente deltaico na área durante essas épocas. Durante o Neocenomaniano; a progradação de sedimentos caracterizados pela UPD, acima da plataforma carbonática do Eo-Mesocenomaniano(?), configura um sistema regressivo para esse intervalo. Definem-se, assim, dois períodos: um transgressivo (Eo-Mesocenomaniano) e outro progradante (Neocenomaniano). Os depósitos deltaicos, abrangendo essas duas fases, ter-se-iam desenvolvido durante todo o Cenomaniano em parte da área estudada (poços 1-PT-1 a 1-RNS-50).

UNIDADE PALEOECOLÓGICA E (UPE) - Durante o periodo transgressivo do Cenomaniano, ocorre a UPE; em camadas com predomínio de calcarenitos. Esses depósitos de plataforma foram acumulados provavelmente até $o$ Mesocenomaniano. A associação microfaunística contém quase exclusivamente Trocholina spp., foraminifero bentôn nico típico de áreas de plataforma carbonática (Dessauvage 1968), escassos ostracodes, entre eles Brachycythere sapucariensis, e o dinoflagelado Cyclonephelium sp. Tibana \& Terra (1981) mencionam algas vermelhas, moluscos e placas de equinóides. Estes autores caracterizam as fácies com todos esses elementos como "barras calcareníticas provavelmente depositadas paralelamente à margem da bacia, em ambiente intermaré e inframaré".

UNIDADE PALEOECOLOGICA F (UPF) - Ainda no Neocenomaniano e abrangendo, possivelmente, o Eoturoniano, na área dos poços 1-RNS-25 e 1-RNS-3; acima da área da plataforma carbonática e, em parte, sobre as fácies deltaicas (poços 3-RNS-38 e 3-RNS-35), está presente uma nova unidade paleoecológica que contém, exclusivamente, foraminíferos planctônicos. São formas globulosas, pustu- 
losas'e sem quilha, em geral pouco diversificadas, mas com grande quantidade de indivíduos. Entre as espécies presentes, encontram-se Whiteinella aprica, $W$. baltica, $W$. brittonensis, $W$. archaeocretacea, Hedbergela ex. gr. delrioensis, H. simplex e Globigerinelloides bentonensis. Entre as formas bisseriadas, aparecem Heterohelix globulosa, $H$. moremani e outros heterohelicídeos pouco desenvolvidos.

Toda esta microfauna caracteriza um ambiente de plataforma média a profunda. As formas que a integram são de hábito planctônico, epipelágicas, que completam seu ciclo de vida nas camadas superficiais dos oceanos e, portanto, são capazes de habitar até as áreas rasas da plataforma.

Segundo Douglas \& Savin (1978), Hart \& Bailey (1979) e Wonders (1980), associações de abundantes hedbergelí. deos e heterohelicídeos, pouco diversificados, caracterizam o começo de ingressões marinhas. Outros autores, entre eles Butt (1982), na área de Marrocos, relacionam este tipo de formas planctônicas com a irrupção de águas frias, com elevada produtividade biológica, nas áreas de plataforma.

$\mathrm{Na}$ área estudada, encontra-se um predomínio de hedber. gelídeos e heterohelicídeos de tamanho reduzido, na base da unidade analisada, enquanto no topo desaparecem os segundos e os hedbergelídeos estão bem desenvolvidos. Considera-se esta modificação na associação microfaunística como produto de um pulso transgressivo que invade áreas de plataforma rasa e sofre um paulatino afundamento, seja por subsidência, ou por variação relativa do nível do mar.

Uma outra característica importante da associação micropaleontológica considerada é a ausência total de fauna bentônica. Este fato indicaria uma anomalia de tipo anóxico somente nas condições do fundo do mar, produzida por uma expansão de um nível de baixa concentração de oxigê. nio nas águas da plataforma, segundo o modelo proposto por Árthur \&Schalanger (1979), ou pela influência exercida por uma elevada taxa de sedimentação clástica, rica em matéria orgânica proveniente das áreas marginais, que con. tribuiria para reduzir as possibilidades de vida bentônica.

Turoniano UNIDADE PALEOECOLOGICA G (UPG) - Acima das unidades paleoecológicas D e F, durante 0 Turoniano, e em uma pequena área até o Campaniano (4-RNS-35), ocorre uma associação que contém ostracodes, restos de equinóides, tubos de vermes, gastrópodes, algas, escassos foraminíferos planctônicos e uma flora dominante de tricolporados lisos e ornamentados, de pequeno porte. Nas camadas turonianas desta unidade, desa" parecem Gonyaulax sp., Cyclonephelium sp., Florentinia sp. e há alta freqüência de dinoflagelados de espinhos curtos, além de uma forma típica do Turoniano denominada informalmente Dinoflagelado $X$. Encontra-se aqui representado o fim de toda uma associação de dinoflagelados indicadores de condiçóes marinhas de águas rasas e movimentadas.

Em camadas correspondentes a esta unidade, Tibana \& Terra (1981) mencionam a presença de miliolídeos, algas verdes e vermelhas, fragmentos de rudistas, interpretando-os como indicadores de plataforma carbonática de pouca profundidade. Tais autores sugerem ainda a existência de fácies de planície de maré e laguna rasa em algumas áreas.

A assembléia polínica, por outro lado - já destituída de Triorites africaensis, Pemphixipollenites inequiexinius, Elateroplicites africaensis, Gnetaceapollenites similis, $G$. classipoli e do gênero Classopollis, que se extinguiram na transição Cenomaniano-Turoniano -, constitui-se predomi- nantemente de angiospermas (tricolporados e tricolpados de pequeno porte). Tal fato mostra que, no tempo de deposição da UPG, acontece o encerramento de um ciclo climá tico árido e quente representado pelas gnetáleas de gxande porte que predominaram durante o Cenomaniano (Gnetaceaepollenites similis, $G$. crassipoli).

Toda esta modificação do espectro polínico pode res. ponder também a uma transgressão muito ampla acontecida na transição Cenomaniano-Turoniano, que mudou as condições ambientais das bacias de margem equatorial brasileira.

Coniaciano-Santoniano Acima dos depósitos que contêm a unidade paleoecológica $\mathrm{G}$, na área dos poços 1.PT-1 e 1-RNS-38, seguem dois intervalos sedimentares represen. tados por calcários. $O$ intervalo inferior é praticamente estéril (é considerado intervalo estéril na figura 3), no qual ocorrem apenas alguns dinoflagelados de espinhos curtos, tecas quitinosas de foraminiferos, além de alguns grãos de pólen. $\mathrm{O}$ superior corresponde à unidade paleoecológica $\mathrm{H}$. $\mathrm{Na}$ área dos poços 4-RNS-35 e 1-RNS-25, as camadas estéreis mencionadas são substituídas lateralmente por calcários de conteúdo micropaleontológico semelhante ao da unidade paleoecológica $\mathrm{G}$.

As condiçóes de deposição de todo o intervalo de idade Coniaciano-Santoniano teriam sido bem mais marginais $e$ restritas que nos intervalos anteriores, pelo menos na área dos poços 1-PT-1 a 1-RNS-38.

UNIDADE PALEOECOLOGICA H (UPH) - Com este intervalo termina $\mathrm{o}$ ciclo carbonático cretáceo na área estudada. Nele está representada uma microfauna bentônica calcário-hialina muito especializada, composta por Buliminella spp., Siphogenerinoides dentata, $S$. clavata, $S$. bramletti, Afrobolivina afra e Lenticulina spp. Relacionada com os foraminíferos, aparece uma associação também especializada de dinoflagelados (Dinogymnium nelsonense, $D$. undulatum e Deflandrea sp.) acrescida de Azolla sp. Esta associação é encontrada nos poços 1-PT-1 a 1-RNS-38 e indicaria um ambiente nerítico raso submetido a influências de aporte de água doce, o que é sugerido pela presença de Azolla sp. (pequena planta aquática de margem de rios). Por outro lado, a ocorrência de associaçðes de buliminídeos e siphogenerinídeos de baixa diversificação, como os detectados na UPH, segundo Butt (1982), leva a pensar em áreas com instabilidade salínica, indicativas de circulação restrita. $\mathrm{Na}$ Bacia de Santos, em áreas de plataforma sob a influência de regime fluvial, também foram identificadas associações pouco diversificadas contendo os mesmos gêneros de foraminíferos (Viviers et al. 1986).

Campaniano - Eomaastrichtiano? Os niveis desta idade estão representados por sedimentos calcários de plataforma e em siliciclásticos de água profunda. Estes últimos estão restritos à área do poço 1-RNS-3.

Os níveis calcários estão restritos ao poço 4-RNS-35 e contêm uma associação micropaleontológica semelhante à da UPG.

UNIDADE PALEOECOLOGICA I (UPI) - Esta unidade é representada por sedimentos clásticos finos, de ambiente de talude, e se restringem à parte mais externa da bacia, a partir do poço 1-RNS-3, na área estudada.

A associação de foraminíferos é formada por uma fauna planctônica bastante diversificada de formas bicarenadas e uma fauna bentônica bem desenvolvida, com alta proporção 
de aglutinantes (Rzehakina sp., Bathysiphon sp., Ammodiscus sp.), característicos de ambientes batiais.

\section{FLuTuAÇóes Relativas do Nivel do MAR -} HIATOS $\mathrm{Na}$ ausência de uma análise bioestratigráfica mais detalhada para o intervalo inferior (Alagoas), iniciarse esta discussão a partir do Albiano.

A primeira elevação relativa do nível do mar (oscilação positiva) é representada por camadas do Albiano, tempo em que se desenvolveu uma plataforma carbonática pouco expressiva. A falta de palinomorfos representativos da Zona Elaterosporites protensus (parte alta do Albiano Superior) e de planctônicos da Zona Ticinella primula indica que o registro Albiano não é completo, tendo-se a falta de, pelo menos, parte do Neoalbiano, o que poderia estar relaciona. do com uma queda relativa do nível do mar.

A partir do Eocenomaniano, ocorreu nova oscilação positiva, que atinge seu clímax no Eo-Mesocenomaniano tendo-se desenvolvido, durante todo este tempo, uma plataforma carbonática extensa. Esta plataforma transgrediu, em direção ao sul, os limites da primitiva plataforma do Albiano. Este episódio é seguido, no Neocenomaniano, de uma queda relativa do nível marinho, de pouca envergadura, indicado pelas relações progradantes de uma associação de foraminíferos, de ambiente marginal, acima da plataforma carbonática, e pelo hiato identificado na área dos poços 1-PT-1 e 1-RNS-50, onde falta o Neocenomaniano.

$\mathrm{Na}$ transição Cenomaniano-Turoniano, representada exclusivamente por depósitos clásticos finos de plataforma, ocorre um terceiro ciclo de oscilação positiva, Tal efeito foi acompanhado de uma evidente modificação faunística, como conseqüiência de variações sedimentares e paleobatimétricas importantes. Passaram a predominar associaçôes formadas exclusivamente por foraminíferos planctônicos. Além de representar uma fase de mar alto, a total ausência de fauna bentônica poderia indicar um período com indícios de anoxia, o qual é síncrono com episódios similares acontecidos em outras áreas, como registram Hart (1980), Jenkins (1980), Wonders (1980) e Seiglie \& Baker (1982).

Ao final do Turoniano, começa a terceira fase de oscilação negativa, evidenciada pela ocorrência de associações microfaunísticas de águas rasas acima de faunas pelágicas. Esta queda relativa do nível do mar prolongou-se pelo Coniaciano-Santoniano, no qual foi mais acentuada. Durante todo este intervalo de tempo, foram depositados carbonatos de plataforma rasa.

Um quarto período de oscilação positiva, no topo do Santoniano, determinou nova deposição de carbonatos de plataforma rasa, que recobriram os sedimentos proximais da plataforma do Turoniano-Santoniano. A tal evento seguiu-se uma nova etapa de oscilação negativa, sugerida pela descontinuidade registrada acima dos sedimentos santonianos.

Finalmente, sedimentos de idade campaniana foram identificados somente na área mais distal da seção estu- dada, os quais foram depositados em ambiente de plataforma (1-RNS-35) e talude (1-RNS-3). Esses depósitos correspondem a um novo e último período de variação do nível do mar, registrado para o Cretáceo da área estudada.

CONCLUSÓES A combinação de dados obtịdos a partir da investigação do conteúdo de foraminiferos e de palinomorfos presentes nos sedimentos cretáceos (Alagoas - Eomaastrichtiano) da Bacia Potiguar permitiu o reconhecimento da evolução bioestratigráfica e paleoecológica desta bacia, na área dos poços 1-PT-1, 1-RNS-36, 1-RNS-50, 3-RNS-38, 4-RNS-35, 1-RNS-25 e 1-RNS-3.

Durante o tempo Alagoas, a sedimentação ocorreu em ambiente continental, e, na fase Albiano/Eomaastrichtiano, os sedimentos depositaram-se sob condições marinhas. Todo o intervalo estudado foi subdivido em 10 unidades paleoecológicas, definidas com base em diferentes padrões de associações fossilíferas. Tais unidades refletem as variações paleoambientais ocorridas na geo-história da área investigada.

Em síntese, a área iniciou sua história com sedimentação flúvio-lacustre e evoluiu para uma sedimentação marinha restrita iniciada em tempos albianos. Sempre sob condições neríticas rasas, porém cada vez mais abertas, esta área da bacia alcançou o Eomaastrichtiano. Por outro lado, durante o transcurso do Cenomaniano, ocorreu uma mudança muito evidente no conteúdo polínico. Desapareceram paulatinamente as efedrales e os elaterosporados, dando lugar, a partir do Turoniano, a uma flora dominada amplamente pelas angiospermas. Esta alteração do espectro polínico evidencia que variações climáticas aconteceram na área: o clima árido e seco tornou-se mais úmido a partir do Turoniano.

É de destacar que, durante o Neocenomaniano -Eoturoniano, parecem ter ocorrido episódios anóxi$\cos$, o que é sugerido pela ausência total de fauna bentônica em depósitos sedimentares de siliciclásticos finos. Tal episódio pode ser paralelo aos já detectados em grande parte das bacias cretáceas atlânticas.

Descontinuidades no registro sedimentar são evidenciadas pela falta de biozonas, indicando hiatos erosivos ou não-deposicionais. Os mais evidentes, relacionados com variações relativas do nível do mar, foram registrados no Neoalbiano, Neocenomaniano e Neocampaniano-Maastrichtiano, todos estes na área mais proximal da bacia. $\mathrm{Na}$ parte mais distal da área estudada, os hiatos mais expressivos abrangem o Coniaciano-Santoniano e o Maastrichtiano. Como resultado deste estudo, é apresentada, também uma curva das variações locais relativas do nivel do mar.

Agradecimentos Os autores agradecem à diretoria executiva da Petrobrás, por permitir a apresentação do trabalho e sua publicação; e ao geólogo D. Dias-Brito, pela leitura crítica do manuscrito.

\section{REFERENNCIAS BIBLIOGRÁFICAS}

ARNAUD-VANNEAU, A. \& PRESTAT, B - - 1984 - Thomasinella and Co. In: SIMPOSIO INTERNACIONAL SOBRE FORAMINÍFEROS BENTICOS, 2, Pau, 1983. Bentho's 83. Paris, Elf-Aquitaine, p. 19-26.
ARTHUR, M.A. \& SCHALANGER, S.O. - 1979 - Cretaceous "oceanic anoxic events" as causual factors in development of reef-reservoired giant oil fields. Am. Assoc. Petr. Geol. Bull., 63(6):870-885. 
BUTT, A. - 1982 - Micropaleontological bathymetry of the Cretaceous of Western Morocco. Paleogeogr., Paleoclimat., Paleoecol., 37(2/4):235-276.

DESSAUVAGE, T.F. - 1968 - Cenomanian Trocholina from Nigeria. Micropaleontology, 14(1):64-72.

DIAS-BRITO, D. - 1985 - Calcisphaerulidae do Albiano da Bacia Potiguar. Considerações paleoecológicas e bioestratigráficas. In: Brasil - MME-DNPM. Col. Trab. Paleont. Brasília. Geologia; 27; Paleontologia e Estratigrafia, 2:307-314.

DOUGLAS, R.G. \& SAVIN, S.M. - 1978 - Oxigen isotopic evidence for the deep stratification of Tertiary and Cretaceous planktonic foraminifera. Mar. Micropaleont., 3:175-196.

EICHER, D.L. - 1969 - Cenomanian and Turonian planktonic foraminifera from the western interior of the United States. In: CONFERÊNCIA INTERNACIONAL SOBRE MICROFOSSEIS PLANCTÔNICOS, 1, Genebra. Proceedings..., Leiden E.J. BULL., p. 137-174.

EICHER, D.L. \& WORSTELL, P. - 1970 - Cenomanian and Turonian foraminifera from the Great Plains, United States. Micropaleontology, 16(3):269-324.

GARGOURI, S. - 1982 - Repartition géografique de Thomasinella punica et d'autres foraminifères cenomaniens. Bull. Soc. Geol. Fr., 24(5/6):943-949.

HARLAND, W.B.; COX, A.V.; LEWELLYN, P.G.; PICKTON, C.A.G.; SMITH, A.G. - $1982-$ A geological Time scale. Cambridge, Cambridge University Press. 131 p.

HART, M.B. - 1980 - A water depth model for the evaluation of planktonic foraminifera. Nature, 286:252-254.

HART, M.B. \& BAILEY, H.W. - 1979 - The distribution of planktonic foraminifera in the Mid-Cretaceous of NW Europe. Int. Union Geol. Sci. Ser. A, 6:527-542.

HART, M.B.; BAILEY, H.W.; FLECHTER, B.; PRICE, R.; SWIEKI, J. - 1981 - Cretaceous. In: JENKINS, D.J. \& MURRAY, J.W. (ed.). Stratigraphic atlas of fossil foraminifera. British Micropaleontological Society, p. 149-227.

HINTE, J.E. van - 1976 - Cretaceous time scale. Am. Assoc. Petr. Geol. Bull., 60(4):498-516.

HUGHES, N.F. \&MOODY-STUART, S.J.C. - 1967 - Palynological facies and correlation in the English Wealden. Rev. Paleobot. Palynol., 1(1):259-268.

JENKINS, H.C. - 1980 - Cretaceous anoxic events from continents to oceans. J. Geol. Soc. London, 137:171-188.

LEHMANN, R. - 1963 - Étude des Globotruncanites du Crétacé Supérieur de la province de Tarfaya (Maroc Occidental). Notes Mem. Sev. Geol. Marocco, 156:133-179.

PETTERS, S.W. - 1977 - Upper Cretaceous planktonic foraminifera from the subsurface Atlantic Coastal Plain of New Jersey. J. Foraminiferal Res., 7(3):165-185.
REGALI, M.S.P. - 1980 - Palinoestratigrafia da Bacia do Ceará. In: CONGR. BRAS. GEOL., 31, Camboriú, 1980. Anais... Camboriú, SBG, v. 5, p. 3118-3129.

REGALI, M.S.P. \& GONZAGA, S. - 1985 - Palinoestratigrafia da Bacia Potiguar. In: Brasil, MME-DNPM. Col. Trab. Paleont. Brasília. Geologia 27; Paleontologia e Estratigrafia 2:443-460.

REGALI, M.S.P.; UESUGUI, N.; LIMA, E.C. - 1985 - Palinoestratigrafia da Bacia de Barreirinhas. Brasília. In: Brasil, MME-DNPM. Col. Trab. Paleont. Brasília. Geologia 27; Paleontologia e Estratigrafia 2:443-460.

REGALI, M.S.P.; UESUGUI, N.; SANTOS, A.S. - 1974 - Palinologia dos sedimentos Meso-Cenozóicos do Brasil. Bol. Téc., Petrobrás, 17(3):171-191 e 17(4):263-301.

ROBASZINSKI, F. \& CARON, M. (coord.) - 1979 - Atlas de foraminifères planctoniques du Crétacé Moyen (Mer Boreal et Tethys). Paris CNRS, 2 v. Cahiers de Micropaleontologie, I/1979 et II/1979.

SEIGLIE, G.A. \& BAKER, M. - 1982 - Foraminiferal zonation of the Cretaceous of Zaire and Cabinda, West Africa and its geological significance. In:. Studies in continental margin geology. Tulsa, p. 651-658 (AAPG Memoir 34).

SOUZA, S.M. - 1982 - Atualização da litoestratigrafia da Bacia Potiguar. In: CONGR. BRAS. GEOL. 32, Salvador, 1982. Anais... Salvador, SBG. v. 5, p. 2392-2406.

TIBANA, P. \& TERRA, G. - 1981 - Seqüências carbonáticas do Cretáceo na Bacia Potiguar. Bol. Téc. Petrobrás, 24(3):174-183.

VAKHRAMEEV, V.A. - 1981 - Classopollis: Indicator of Jurassic and Cretaceous climates. The Palaeobotanist, (28/29):301-307.

VIVIERS, M.C. - 1982 - Biocronoestratigrafia da Bacia do Ceará In: CONGR. BRAS. GEOL., 32, Salvador, 1982. Anais... Salvador, SBG. v. 5, p. 2433-2449.

VIVIERS, M.C. - 1985 - Características bioestratigráficas dos sedimentos Albo/Cenomanianos da Bacia do Ceará. Relações com outras bacias brasileiras e africanas. In: Brasil, MME-DNPM. Col. Trab. Paleont. Brasília. Geologia 27; Paleontologia e Estratigrafia 2:523-537.

VIVIERS, M.C.; UESUGUI, N.; RICHTER, A.; PRAÇA, U.M.; HASHIMOTO, A.T.; ANTUNES, R.; HERTER, G.G. - 1986 - Bioestratigraphy and paleoecological evolution of the Middle and Upper Cretaceous of the Santos Basin, Brazil. An. Acad. bras. Ciênc., 58(1):172-173.

WONDERS, A.A.H. - 1980 - Middle and Late Cretaceous planktonic foraminifera of the Western Mediterranean area. Utrecht Micropaleontological Bulletin, 24.157 p.

MANUSCRITO 381

Recebido em 01 de setembro de 1986 Revisão aceita em 30 de março de 1987
“... o que realmente torna o intelectualismo uma forma perversa de elitismo é o fato de alienar-se do mundo e servir apenas às minorias privilegiadas. Neste sentido, não é contra a qualidade, não é contra a sofisticação de pensamento que devemos lutar no antielitismo; devemos lutar contra a má qualidade e a má distribuição do produto que geramos na universidade".

C. Buarque, 1986, Pau Brasil, 14: p.72 